\title{
Effects of Recombinant Bovine Granulocyte-Macrophage Colony-Stimulating Factor on Bovine Peripheral Blood Neutrophil Functions in vitro and in vivo
}

\author{
Tsunao HIRAI, Manabu OIKAWA, Shigeki INUMARU'1), Yuichi YOKOMIZO'), Naohito KUSAKARI and
} Kiyokazu MORI

Hokkaido Prefectural Shintoku Animal Husbandry Experiment Station, 4-40 Shintoku-cho, Hokkaido 081-0038 and ${ }^{1 /}$ National Institute of Animal Health, 3-1-1 Kannondai, Tsukuba, Ibaraki 305-0856, Japan

(Received 26 April 1999/Accepted 24 July 1999)

ABSTRACT. Effects of recombinant bovine granulocyte-macrophage colony-stimulating factor (rboGM-CSF) on bactericidal activity of bovine peripheral blood neutrophils in vitro and in vivo were studied. In in vitro experiment, bovine blood neutrophils were cultured for 9 hr in media containing $0.005,0.05$ or $0.5 \mu \mathrm{g} / \mathrm{m} l$ of rboGM-CSF. Neutrophils treated with rboGM-CSF showed significantly higher luminol-dependent chemiluminescence (LDCL) than control cells. In in vivo experiment, neutrophils isolated from cows injected 5.0 $\mu \mathrm{g} /$ $\mathrm{kg}$ of rboGM-CSF showed significantly higher Nitrobluetetrazolium (NBT) reduction value than that from control cows 24 hr post injection. Total leukocyte counts of cows injected rboGM-CSF sharply decreased 6 hr post injection and recovered to normal level 2 days post injection. Body temperature of these cows rose $6 \mathrm{hr}$ post injection and back to normal level at $24 \mathrm{hr}$ post injection. It was suggested that rboGM-CSF enhanced bactericidal activity of bovine neutrophils both in vitro and in vivo.-KEY wORDS: bactericidal activity, neutrophil, recombinant bovine GM-CSF.

Granulocyte-macrophage colony-stimulating factor (GM$\mathrm{CSF})$ is a glycoprotein involved in the proliferation and differentiation of normal haematopoietic cells. This factor is not only regulating the myeloid precursor cell proliferation and differentiation, but also enhancing mature neutrophil, eosinophil and monocyte/macrophage functions $[4,8,9,16$, 18, 24, 29]. Recombinant human GM-CSF (rhuGM-CSF) has been used for treatment of leukopenic patients with aplastic anemia or acquired immunodeficiency syndrome [3, 15, 17]. Reddy et al. [23] and Sordillo et al. [26] reported that bovine neutrophils treated with recombinant bovine (rbo) GM-CSF in vitro showed higher bactericidal activity than non-treated control cells. Neutrophil functions are closely related with outcome of bovine infectious bacterial diseases, such as mastitis [1, 5, 14]. However, bactericidal activity of bovine mammary gland neutrophils which infiltrate from blood was lower than that of peripheral blood neutrophils [7, 21]. In the case of bovine acute clinical mastitis, the bactericidal activity of blood neutrophils decreased at the onset or initial period of inflammation, and increased during recovery [20]. These reports suggested that enhancement of blood neutrophil bactericidal activity by rboGM-CSF may have preventive or therapeutic effect on bovine mastitis. However, only few experiments have been done about in vivo effects of rboGMCSF on neutrophil functions in luminants $[6,12,25]$, and the results were not consistent. In the present study, in vitro and in vivo effects of rboGM-CSF produced in Baculovirus expression system [10] were investigated.

The recombinant virus infected serum free culture fluid was used as the rboGM-CSF source, after removal of viral particles by ultra centrifugation and ultra filtration. Wild type virus infected cell culture fluid was used as placebo.

For in vitro experiment, venous blood samples were collected once from eleven Holstein cows (12-39 weeks after parturition, 2-6 years old). In in vivo experiment, eleven Holstein cows (11-43 weeks after parturition, 2-5 years old) were assigned randomly to three treatment groups of four and three cows. Two groups of four cows received single subcutaneous injection of rboGM-CSF at doses of $5.0 \mu \mathrm{g} / \mathrm{kg}$ body weight and placebo in the cervical region, respectively. One group of three cows received single subcutaneous injection of rboGM-CSF at doses of $2.5 \mu \mathrm{g} /$ $\mathrm{kg}$ body weight. Venous blood samples were collected before the treatment and 6,12,24 hr, 2, 3 and 7 days post injection of rboGM-CSF or placebo.

Neutrophils were isolated from the heparinized blood by Ficoll-Conray density gradient centrifugation [19].

In in vitro experiment, neutrophils isolated from each cow were incubated with Hanks Balanced Salt Solution supplemented with $0.005,0.05$ or $0.5 \mu \mathrm{g} / \mathrm{m} l$ of rboGM-CSF $\left(5 \times 10^{6}\right.$ cells $\left./ \mathrm{ml}\right)$ in 24-well plates (Becton Dickinson and Co., U.S.A.) for $9 \mathrm{hr}$ at $37^{\circ} \mathrm{C}$ in $5 \% \mathrm{CO}_{2}$. Untreated neutrophils served as a control.

Luminol-dependent chemiluminescence (LDCL) assay and Nitrobluetetrazorium (NBT) reduction assay were performed according to the method described previously $[20,28]$ with slight modification in reagent concentration, respectively.

In in vitro experiment, neutrophils treated with 0.05 and $0.5 \mu \mathrm{g} / \mathrm{m} l$ of rboGM-CSF showed higher peak LDCL response from start (just after treatment) to $9 \mathrm{hr}$ after treatment than untreated control cells. Neutrophils treated with $0.005 \mu \mathrm{g} / \mathrm{m} l$ of rboGM-CSF showed less significant, however only just after treatment, neutrophil activities showed statistically higher value than control (Table 1).

In in vivo experiment, positive reactions of NBT reduction had been seen until 2 days post injection by all three groups. Among them, the values of $5.0 \mu \mathrm{g} / \mathrm{kg}$ injected cows were significantly high (Fig. 1). However, peak time was varied among four cows in this group, i.e. $6 \mathrm{hr}$ post injection in two cows, $12 \mathrm{hr}$ in one cow and $24 \mathrm{hr}$ in one cow. At six 
Table 1. Changes in peak LDCL response $\left(\times 10^{6} \mathrm{CPM}\right)$ of neutrophils treated with rboGM-CSF

\begin{tabular}{lcccc}
\hline \multirow{2}{*}{$\begin{array}{l}\text { GM-CSF concentration } \\
(\mu \mathrm{g} / \mathrm{m} l)\end{array}$} & \multicolumn{5}{c}{ Incubation time (hours) } \\
\cline { 2 - 5 } & 0 & 3 & 6 & 9 \\
\hline \multirow{2}{*}{$0.5(\mathrm{n}=11)$} & $3.863^{*}$ & $4.644^{*}$ & $4.972^{* *}$ & $5.187^{*}$ \\
& $(0.612)$ & $(0.978)$ & $(0.990)$ & $(1.013)$ \\
$0.05(\mathrm{n}=11)$ & $3.859^{* *}$ & $4.467^{*}$ & $4.840^{*}$ & $5.098^{*}$ \\
& $(0.535)$ & $(0.937)$ & $(1.018)$ & $(1.131)$ \\
$0.005(\mathrm{n}=9)$ & $3.966^{* *}$ & 4.395 & 4.809 & 5.002 \\
& $(0.683)$ & $(0.993)$ & $(1.004)$ & $(1.178)$ \\
$0.0(\mathrm{n}=11)$ & 3.321 & 3.873 & 4.095 & 4.423 \\
& $(0.709)$ & $(0.782)$ & $(0.697)$ & $(0.583)$ \\
\hline
\end{tabular}

Each value in parenthesis is SD. Higher value compared with control $(0.0 \mu \mathrm{g} / \mathrm{m} l$ of GM-CSF concentration) in each incubation time by the $t$-test $(* \mathrm{P}<0.05, * * \mathrm{P}<0.01)$

hours post injection, total leukocyte counts in peripheral blood from cows injected with 5.0 or $2.5 \mu \mathrm{g} / \mathrm{kg}$ of rboGMCSF decreased significantly (data not shown). Body temperature of these cows rose until $24 \mathrm{hr}$ (data not shown). Both total leukocyte counts and body temperature were not affected by placebo injection. At sites of injection, no clinical side effects as swelling or induration were observed.

In vitro effects of rboGM-CSF on bovine neutrophil functions were previously reported [23, 25]. In these studies, neutrophils treated with $0.005 \mu \mathrm{g} / \mathrm{m} l$ of rboGMCSF for 9 to $12 \mathrm{hr}$ showed significantly higher phagocyting or bactericidal activities than control cells. In the present study, neutrophils treated with $0.5 \mu \mathrm{g} / \mathrm{m} l$ of rboGM-CSF showed the highest peak LDCL response among 0.005, 0.05 and $0.5 \mu \mathrm{g} / \mathrm{m} l$ in culture medium. Sordillo et al. [26] reported that bactericidal activity of neutrophils treated with $0.005 \mu \mathrm{g} / \mathrm{m} l$ of rboGM-CSF was not significantly higher than that of control cells until $9 \mathrm{hr}$ of incubation. In the present study, bactericidal activity of neutrophils treated with rboGM-CSF was significantly higher than control cells just after incubation. These differences may be due to the differences of the recombinant cytokines. Former works have been done with E. coli expressed rboGM-CSF, on the other hand, our works have been done with Baculovirus expressed one. There are some differences of posttranslational modification between them. There may be some quantitative differences of biological activity as well. In vivo effects of rboGM-CSF on neutrophil functions in cattle were rarely reported. Daley et al. [6] showed that intramammary infusion of rboGM-CSF resulted in enhancement of inducible oxygen radical formation and phagocytosis in milk neutrophils. Kehrli [12] reported that no enhancement of various neutrophil or lymphocyte functional activities was detected with repeated systemic administration of rboGM-CSF. On the other hand, Sordillo [25] reported enhancement of NBT reduction value of neutrophils in goats.

In the present study, it was demonstrated that bactericidal activity of peripheral blood neutrophils was enhanced with

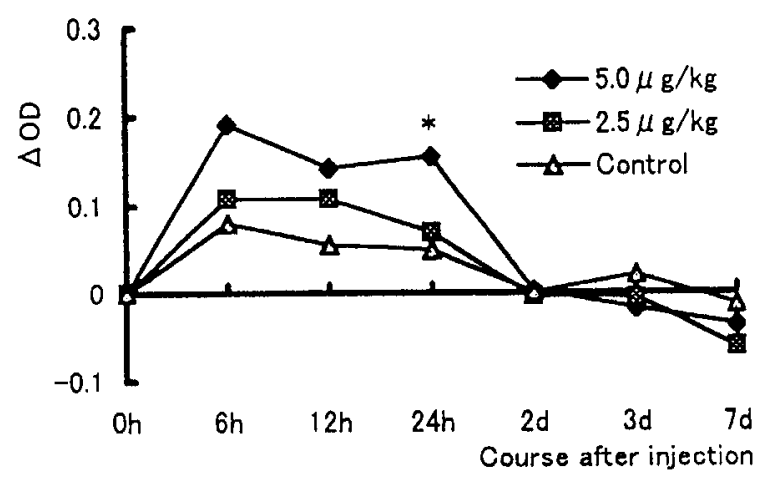

Fig. 1. NBT reduction value post injection of rboGM-CSF. *: $\mathrm{P}<0.05$ significant difference compared with control value by the $t$-test.

subcutaneous injection of rboGM-CSF. This result suggested the possibility of rboGM-CSF for clinical use to protect or treat bacterial infections such as mastitis in cattle. Dulin et al. [7] and Nickerson [21] demonstrated that phagocytic and bactericidal activities of bovine mammary gland neutrophils were lower than those of blood neutrophils due to lack of an energy source, decrease in opsonins and interference by casein and fat. It makes possible to survive some kind of bacteria as Staphylococcus aureus (S. aureus) in these cells after phagocytizing $[1,2]$. Lohuis et al. [14] showed that preinfection bactericidal activity of blood neutrophils was negatively correlated with counts of $E$. coli in milk and with losses in milk production in experimental E. coli mastitis. Enhancement of neutrophil bactericidal activity is indispensable to prevent or treat infectious diseases, especially bovine mastitis. On the other hand, side effects of rboGM-CSF must be noticed. In this study, transient decrease in blood leukocyte counts and fever were observed by administration of rboGM-CSF. These phenomena were also observed in previous reports $[11,12$, $25,27]$. Decrease in blood leukocyte counts may be due to the margination of neutrophils, because it was reported that rhuGM-CSF alter the expression of adhesive receptors on phagocytes [30].

Our results in this report, together with data of other groups, indicated that rboGM-CSF is one of the good candidate for therapeutic agent in cattle, though the side effects i.e. elevation of body temperature was observed.

In case of recombinant granulocyte colony-stimulating factor (G-CSF), some promising results for the protective effects against bovine bacterial infections were reported [13, 22].

Therefore, comparative studies of rboGM-CSF and rboGCSF may required to elucidate protective efficacy against bovine bacterial diseases and side effects.

ACKNOWLEDGMENTS. This work was supported in part by a grant-in-aid (Recombinant Cytokine Project) from Ministry of Agriculture, Forestry and Fisheries of Japan 
(RCP1999-1120). We thank Dr. H. Nagahata (College of Dairying, Rakuno Gakuen), Dr. S. Araki (Eisai Co., Ltd) and Dr. H. Takahashi (National Institute of Animal Health) for advising on NBT reduction assay or LDCL assay. We also thank Dr. S. Onoe (Hokkaido Prefectural Takikawa Animal Husbandry experiment station) for statistical analysis, and Mr. K. Okahashi, Mr. S. Yoshida and Mr. M. Murota (Berthold Japan Co., Ltd.) for according facility for using multi channel luminometer.

\section{REFERENCES}

1. Anderson, J. C. 1982. Vet. Rec. 110: 372-376.

2. Anderson, J. C. and Williams, M. R. 1985. J. Med. Microbiol. 20: 317-323

3. Baldwin, G. C., Gasson, J. C., Quan, S. G., Fleischmann, J., Weisbart, R., Oette, D., Mitsuyasu, R. T. and Golde, D. W. 1988. Proc. Natl. Acad. Sci. 85: 2763-2766.

4. Clark, S. C. and Kamen, R. 1987. Science 236: 1229-1237.

5. Cooray, R. and Håkannson, L. 1995. J. Vet. Med. B 42: 625632.

6. Daley, M., Williams, T., Coyle, P., Furda, G., Dougherty, R. and Hayes, P. 1993. Cytokine 5: 276-284.

7. Dulin, A. M., Paape, M. J. and Nickerson, S. C. 1988. Am. J. Vet. Res. 49: 172-177.

8. Fleischmann, J., Golde, D. W., Weisbart, R. H. and Gasson, J. C. 1986. Blood 68: 708-711.

9. Gasson, J. C., Weisbart, R. H., Kaufman, S. E., Clark, S.C., Hewick, R.M., Wong, G. G. and Golde D.W. 1984. Science 226: 1339-1342.

10. Inumaru, S., Kokuho, T., Mori, S., Watanabe, S. and Takamatsu, H. 1997. Cytokine 9: 901.

11. Kasakura, S. 1991. Cytokine. p. 90. Nihon Igakukan, Tokyo (in Japanese).

12. Kehrli, M. E. 1993. J. Leukocyte Biol. 54 (Suppl.): 60.

13. Kehrli, M. E., Goff, J. P., Stevens, M. G., Thomas, C. and Boone, T. C. 1991. J. Dairy Sci. 74: 2448-2458.
14. Lohuis, J. A. C. M., Schukken, Y. H., Henricks, P. A. J., Heyneman, R., Burvenich, C., Verheijden, J. H. M., Van Miert, A. S. J. P. A. M. and Brand, A. 1990. J. Dairy Sci. 73: 342350.

15. Lopez, K. D. and Guinan, E. C. 1995. Pediatr. Nurs. 21: 345-349.

16. Lopez, A. F., Williamson, D. J., Gamble, J. R., Glenn Begley, C., Harlan, J. M., Klebanoff, S. J., Waltersdorph, A., Wong, G., Clark, S. C. and Vadas, M. A. 1986. J. Clin. Invest. 78: 1220-1228.

17. Manfredi, R., Mastroianni, A., Coronado, O. and Chiodo, F. 1996. J. Chemother. 8: 214-220.

18. Metcalf, D. 1985. Science 229: 16-22.

19. Nagahata, H., Noda, H. and Kudo, T. 1982. Jpn. J. Vet. Sci. 44: 957-960.

20. Nagahata, H., Yatsu, A. and Noda, H. 1986. Br. Vet. J. 142: 578-584.

21. Nickerson, S. C. 1985. J. Am. Vet. Med. Assoc. 187: 41-45.

22. Nickerson, S. C., Owens, W. E. and Watts, J. L. 1989. J. Dairy Sci. 72: 3286-3294.

23. Reddy, P. G., McVey, D. S., Chengappa, M. M., Brecha, F., Minocha, H. C. and Baker, P. E. 1990. Am. J. Vet. Res. 51: 1395-1399.

24. Smith, P. D., Lamerson, C. L., Wong, H. L., Wahl, L. M. and Wahl, S. M. 1990. J. Immunol. 144: 3829-3834.

25. Sordillo, L. M. 1992. Proc. 31st Annu. Mtg. Natl. Mastitis Counc.: 160-164.

26. Sordillo, L. M., Afseth, G., Davies, G. and Babiuk, A. 1992. Can. J. Vet. Res. 56: 16-21.

27. Stern, A. C. and Jones, T. C. 1992. Infection 20 (Suppl. 2): 124-127.

28. Takahashi, H. and Miyamoto, T. 1991. Res. Rep. Anim. Health No. 5: 29-30 (in Japanese).

29. Weisbart, R. H., Golde, D. W., Clark, S. C., Wong, G. G. and Gasson, J. C. 1985. Nature (Lond.) 314: 361-363.

30. Yong, K. I. and Linch, D. C. 1992. Eur. J. Haematol. 49: 251-259. 